\title{
Dual-balloon assisted super-selective embolisation of high flow arterial venous fistula within a transplant kidney
}

\author{
Sara ffrench-Constant ${ }^{1 *}$ D, Nisal Weerakoon², Rahul Amin², Luke Dixon', David Taube ${ }^{3}$ and Mohamad Hamady ${ }^{1}$
}

\begin{abstract}
Background: In this case report, we describe a novel application of the technique of 'dual-balloon assisted' cannulation and embolisation of a high flow arterial venous fistula (AVF) in transplant kidney, where attempts at standard and previously described embolisation techniques were proving difficult to achieve.

Case presentation: Seventy year old gentleman with renal transplant presenting with high output cardiac failure and deteriorating renal function. Angiography demonstrated high flow traumatic AV fistula within transplanted kidney, secondary to multiple biopsies. Attempts at guidewire and guiding sheath placement and stability for occlusion plug deployment were failing due to combination of very high back-flow pressures within the AVF and challenging vascular anatomy; with an aneurysmal, tortuous iliac artery as well as intra renal transplanted vessels. A combination of angioplasty and remodeling aortic balloons in the transplant artery and the host external iliac vein respectively, facilitated stabilization of guiding sheath and hence controlled delivery of an occlusion plug from the venous side of the fistula. The fistula was successfully embolised, leading to complete resolution of patient symptoms and improvement of renal function beyond his previous baseline.

Conclusions: Percutaneous embolisation is an established technique to treat iatrogenic AVF in transplant kidneys. High flow pressure through an AVF, as demonstrated in this case, can cause difficulty and raise safety issues in accessing and embolising the AVF using previously described techniques. This case report describes an effective and novel application of the technique of using a second balloon in the host common iliac vein to; lower flow pressure, stabilise the guidewires during plug deployment and prevent displacement of wires and/or plug into the common iliac vein.
\end{abstract}

Keywords: Embolisation, Balloon assisted embolisation, latrogenic fistula, Renal transplant, High flow arteriovenous fistula

\section{Background}

An arteriovenous malformation is an abnormal communication between arterial and venous systems. An acquired communication, as opposed to a developmental one, is an arteriovenous fistula (AVF) (Eibl et al. 2009). The incidence of intrarenal AVMs is fairly low, estimated to be around $0.3 \%$ in native kidneys. However, the incidence of AVFs is much higher, especially in transplant kidneys - closer to 6$8 \%$ (Vij et al. 2011). AVFs have long been a recognized complication post renal transplant biopsy (Merkus et al. 1993;

\footnotetext{
* Correspondence: sara.ffrench-constant@nhs.net

${ }^{1}$ Imperial College Healthcare Trust, London, UK

Full list of author information is available at the end of the article
}

Diaz-Buxo et al. 1974). Whilst these are often quiescent, if left untreated, they can lead to deterioration in renal function and high out-flow cardiac failure (Maldonado et al. 1964). Once symptomatic, an AVF requires treatment - of which embolization is the favoured technique. This can be achieved by a number of minimally invasive transcatheter methods, including occlusion plug embolization (Rajesh et al. 2015) and coil embolization (Sundarakumar et al. 2013). High flow in the fistula usually imposes a significant safety challenge during intervention. This case describes a novel application of the technique of dual balloon occlusions facilitating plug embolisation from the venous side of the fistula. Ethical approval was obtained according to our institutional guidelines. 


\section{Case presentation}

We present a case of a 70-year old Caucasian gentleman who underwent a living donor renal transplant 10 years ago following a diagnosis of glomerulonephritis aged 34. More recently, he had developed acute heart failure manifested by bilateral leg oedema, facial oedema and difficulty in breathing together with severe and difficult to control hypertension with wide pulse pressures. Biochemistry at the time of presentation revealed Cr $190 \mathrm{mmol} / \mathrm{L}$ and eGFR 27-32.

The patient had past history of several transplant biopsies performed to investigate repeated rise in serum creatinine level over the last 10 years. Clinically, there was audible bruit over the right side of the lower abdomen. Ultrasound, CT angiography scan and subsequently catheter angiography demonstrated very high flow arterial venous fistula within the transplanted organ (Fig. 1), likely to be the cause of the patients symptoms of decompensated heart failure. The main transplant artery and veins, as well as all intra renal branches were aneurysmal with extreme tortuosity, especially in the intra renal vessels. The maximum diameter of the main transplant artery was $12 \mathrm{~mm}$ with relative narrowing at the origin. Other salient findings were ectatic and tortuous iliac vessels (Fig. 2). Given the decompensated heart failure being caused as a result of the high flow AVF, patient planned for super-

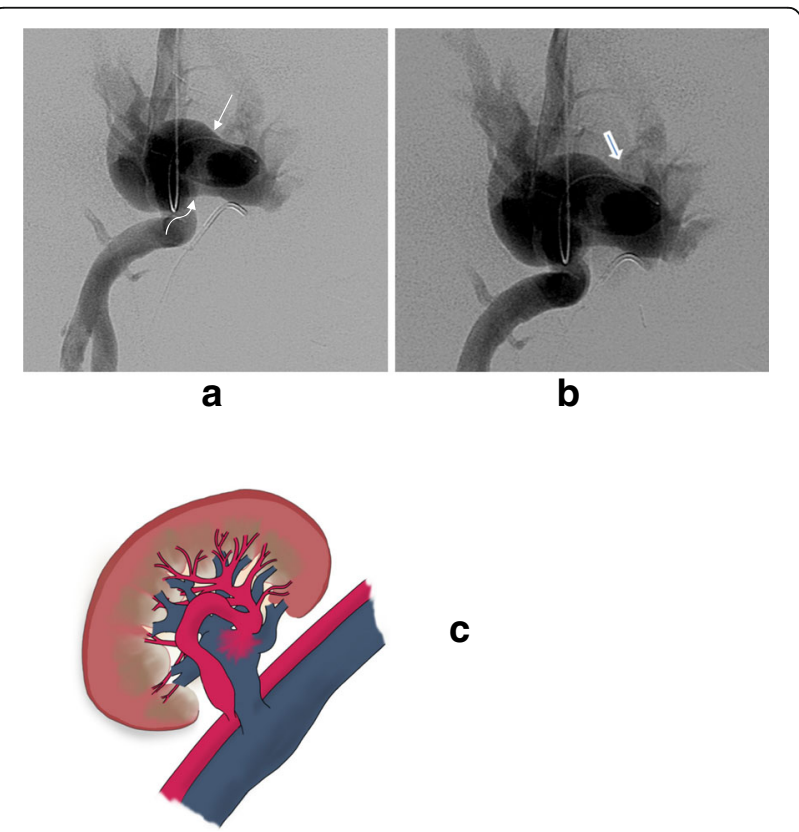

Fig. 1 Angiographic and schematic demonstration of high flow arterio-venous fistula within the transplanted kidney. a Aneurysmal intra renal artery (white arrow) and relative narrowing at the AVF connection (curved arrow) demonstrated on pre-procedure angiography. b Left lateral oblique view shows early draining aneurysmal renal vein (solid arrow). c Schematic diagram demonstrating the high flow AVF within the transplant kidney and highlighting the tortuous vessel anatomy selective embolisation of the arterio-venous communication under interventional radiology.

Informed consent was obtained and patient readmitted for elective super selective renal transplant embolization, to be performed under general anaesthesia in order to control the patient's haemodynamic status. Given the knowledge of high flow through the AVM, plans were made to make both arterial and venous punctures. The contralateral left common femoral artery was punctured under ultrasound guidance and $6 \mathrm{~F}$ sheath was inserted and positioned in the right common iliac artery. Through the sheath, the transplant artery was selected and $6 \mathrm{mmx} 40 \mathrm{~mm}$ angioplasty balloon was positioned and inflated at the origin of the transplant artery to reduce inflow and hence pressure into the AVM. The site of the AVM was approached via the venous side.

Since the arterial side has a tight, almost $360^{\circ}$, backward bend to AV communication site and the venous channel has shorter and straighter path, a $6 \mathrm{~F}$ sheath was inserted into the right common femoral vein, aiming to deliver the embolic device. Multiple attempts at cannulating the fistula from the venous side failed due to the predicted factors previously described. The tortuosity of the vessels led to difficult in reaching the exact site of the AVM, whilst the high flow from the arterial side continually forced the guide wires and catheters out of the transplant vein and back up into the common iliac vein as the inflated balloon at the origin of the artery failed to reduce the flow.

Therefore, an additional $12 \mathrm{~F}$ sheath and balloon (Medtronic Reliant ${ }^{\mathrm{M}}$ stent graft balloon catheter) were inserted into the venous ipsilateral side, coming from an insertion site just above the initial puncture. This balloon allowed transient occlusion of the common iliac vein (Fig. 3a), to ensure the guide wire remained in the transplant vein and not forced back into the host common iliac vein by the arterial pressure coming through the AVM. Once the wire was secured in situ, an occlusion plug (10 mmx7mm) (AMPLATZER ${ }^{\mathrm{Tw}}$ Vascular Plug II, Abbott) was advanced through a $6 \mathrm{~F}$ sheath and deployed across the fistula (Fig. 3b). This led to good embolic occlusive result with subsequent angiography showing no flow across the previous fistula (Fig. 4 - (a) pre occlusion plug and (b\&c) post occlusion plug).

Following successful embolisation of the fistula, patient's haemodynamic status remained stable. There was a transient rise in serum creatinine immediately after the procedure due to contrast nephrotoxicity from the procedure itself. In the proceeding weeks, the blood pressure decreased to around 130/85 systolic and serum creatinine improved to 138 on latest bloods. Of most importance, the patient is clinically significantly improved and has reported complete resolution of breathlessness and oedema - suggesting his symptoms were a direct result of the AVF causing high flow cardiac failure.

USS of the transplanted kidney performed 1 week after intervention showed good perfusion and no evidence of 


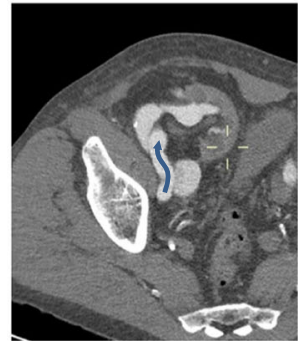

a

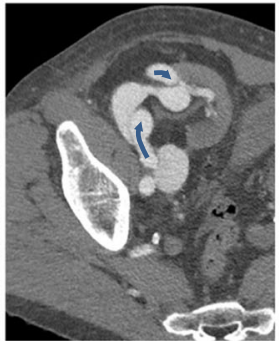

b

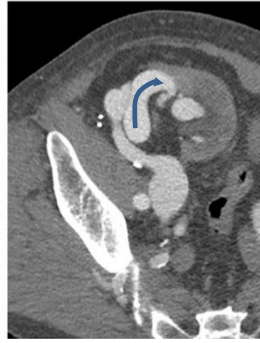

C

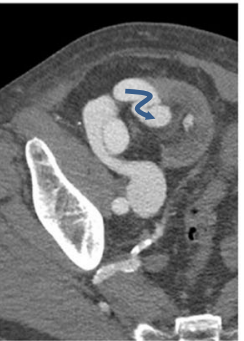

d

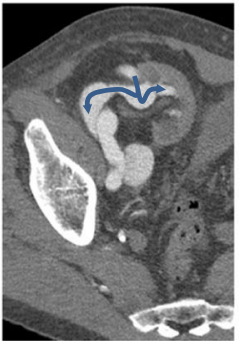

e

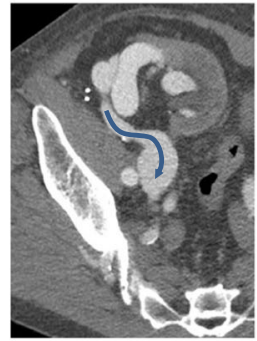

f

Fig. 2 Series of CTA images demonstrating high flow arterio-venous fistula within the transplanted kidney. Block arrow demonstrating route of blood from transplant artery (images a-d), the AV fistula (image e) and the early filling of the transplant renal vein (image $\mathbf{f}$ )

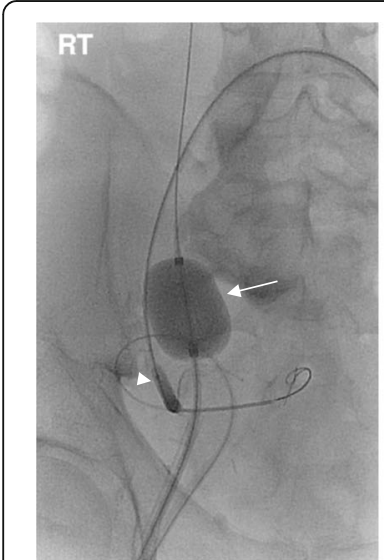

a

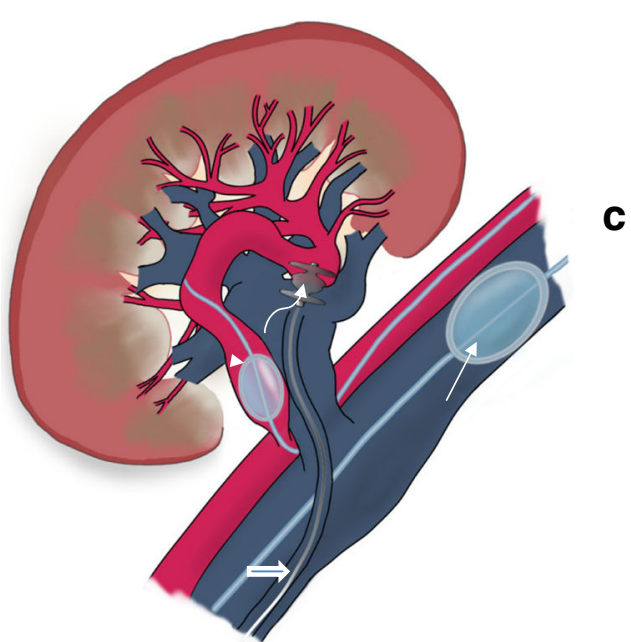

Fig. 3 Super-selective embolisation of the AVF. a and bTransient balloon occlusion of the artery (arrow head) and common iliac vein (white arrow) to allow advancement of the sheath (solid arrow) and deployment of Amplatzer plug (curved arrow) across AVF. c Schematic diagram demonstrating the above embolisation technique a residual or recurrent AV fistula. Follow up CT Angiogram performed 2 months after procedure confirmed good perfusion of transplanted kidney, stable position of the occlusion device at AVF site and resolution of the previous AVF (Fig. 5).

\section{Discussion}

Angiographic embolization of arteriovenous fistulae is a successful treatment in patients with renal transplant AVF and a technique that has been well described in the literature. The benefit of this therapy is highlighted in a retrospective review of embolic therapy utilizing a super-selective technique. Twenty-one renal transplant patients with iatrogenic AVF's were reviewed, with successful embolisation demonstrated in $95 \%$ of cases with no serious complications (Perini et al. 1998). Effective therapy led to eradication of main presenting symptom in $88 \%$ of patients with $58 \%$ demonstrating no detrimental effect on transplant function long-term. A similar case series followed selective embolisation of 10 renal transplant AVFs secondary to renal biopsy (Schwarz et al. 2008). These 10 cases were from an initial cohort of 2824 renal transplant biopsies, with AVFs confirmed by Doppler US in 235 of these (8.3\%). On follow up, only 30 patients had persistent AVFs, with the remaining spontaneously resolving. Of the 10 selective embolisations performed on symptomatic AVFs, 9 were technically successful with no complications reported. Interestingly, improved graft function was only noted in $60 \%$, highlighting the fact that successful embolisation does not always correspond to improved outcome. The remaining case in this review led to unsuccessful closure of the AVF and resulted in a small area of infraction in the transplant parenchyma. A final case review of 13 patients undergoing selective embolisation for symptomatic AVFs post biopsy, demonstrated successful embolisation in all patients (Maleux et al. 2003). Resolution of symptoms was seen in all 13 and improvement in graft function in 10. Only one complication was observed in this study, with thrombosis of a segmental artery, immediately treated with in situ thrombolysis and no long term ill effect. In a summary of the cases described so far, whilst renal 


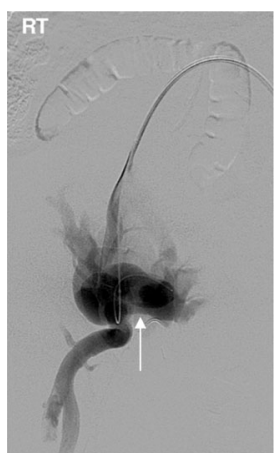

a

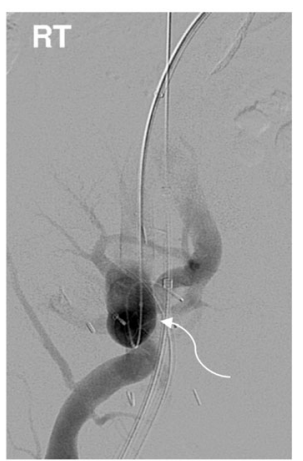

b

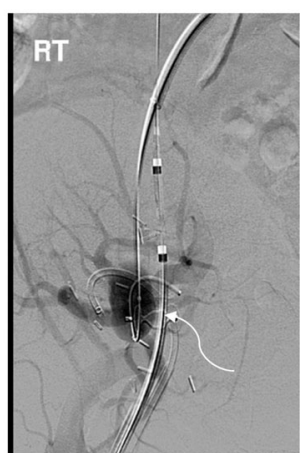

C

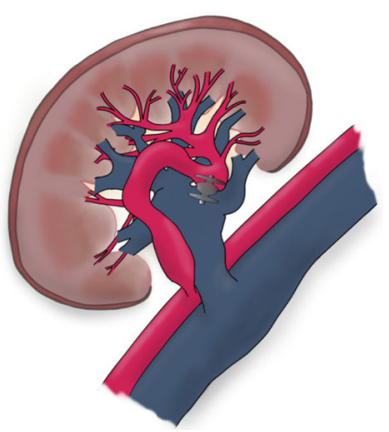

d

Fig. 4 Angiographic demonstration of successful embolisation of AVF. The AVF (white arrow) demonstrated pre (a) and post (b \& c) successful Amplatzer plug (curved arrow) occlusion, with no filling of the fistula. $\mathbf{d}$ Schematic diagram confirming Amplatzer plug occlusion of the AVF with no through flow

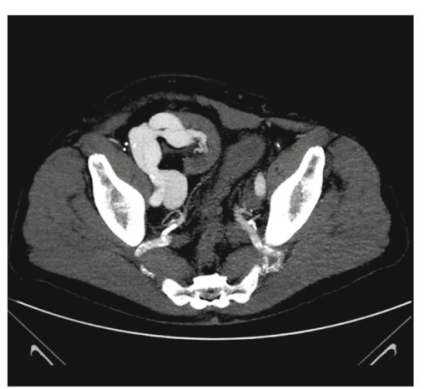

a

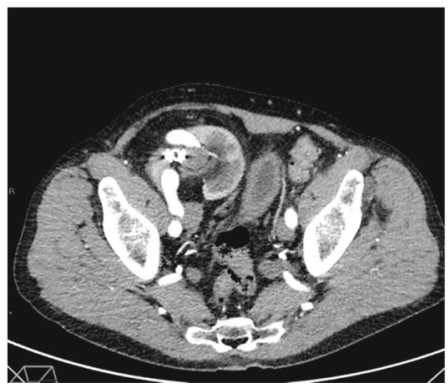

C

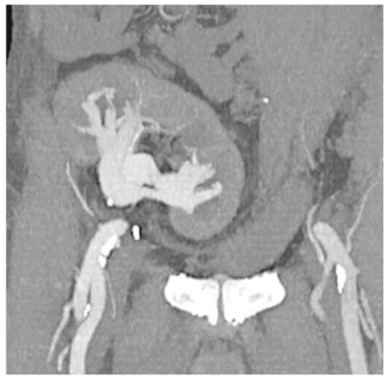

b

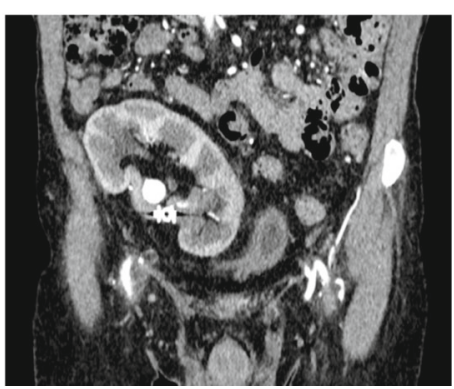

d

Fig. 5 2-month follow up $\subset$ angiogram. Pre procedure axial (a) and coronal (b) and post procedure axial (c) and coronal (d) images prove successful closure of AVF with Amplazter plug and no perfusion defect 
transplant biopsies are performed commonly, the complication of a symptomatic AVF resulting from a biopsy is rare. Despite this, successful embolisation has been reported in the majority of cases, with most of these leading to improved renal transplant function and/or amelioration of the presenting symptom.

In the vast majority of the cases described in the literature, access to the AVF is gained via the arterial side and occlusion achieved with plugs or coils. Dual-balloon assisted embolisation technique has been previously described in the same and in other vascular territories (Shih et al. 2010; Barley et al. 2006). However, in our case report we embolised the fistula from the venous side while two balloons were inflated on either sides of the fistula to combine; reduction in blood flow, facilitate sheath advancement through severe tortuosity and prevent guide wire misplacement as well as occlusion plug migration. We feel this new technique of using a second balloon to stabilize the guide wires and prevent displacement into the common iliac vein can be exploited to aid access in similar cases.

\section{Conclusions}

In conclusion, the occlusion of high flow AVF associated with extreme vessel tortuosity, vessel ectasia and short connecting channel can be achieved using dual occlusion balloons and embolisation via venous approach. The occlusion balloons will not only reduce speed of flow and minimize risk of device dislodgment but also smooth the convoluted path of guiding sheath or catheter to reach the target point.

\section{Abbrevations}

AVF: Arterio-venous fistula; AVM: Arterio-venous malformation; $\mathrm{Cr}$ : Creatinine; $\mathrm{CT}$ : Computed tomography; eGFR: Estimagted glomerular filtration rate; 6F/ 12F: 6 french/12 french; USS: Ultrasounds scan

\section{Acknowledgements}

This study was supported by Imperial College London Healthcare Biomedical Research Centre and the Imperial College London Charitable Trustees Fund.

\section{Availability of data and materials}

Relevant data supporting the findings detailed in this paper can be found within the manuscript itself.

\section{Authors' contributions}

SFC and MH carried out the interventional procedure, conceived of the manuscript and drafted and finalised the submission. NW and RA assisted in draft versions of the manuscript. LD produced all schematic images. DT is patient's clinician and was responsible for patient's management and follow up. All authors have read and approved the final manuscript.

Ethics approval and consent to participate

Ethical approval was obtained according to our institutional guidelines.

\section{Consent for publication}

Informed, written consent from the patient was obtained and consent form signed.

\section{Competing interests}

The authors declare that they have no competing interests.

\section{Publisher's Note}

Springer Nature remains neutral with regard to jurisdictional claims in published maps and institutional affiliations.

\section{Author details}

${ }^{1}$ Imperial College Healthcare Trust, London, UK. ${ }^{2}$ Imperial College London, London, UK. ${ }^{3}$ Imperial College Health Trust, London, UK.

Received: 30 May 2018 Accepted: 14 September 2018

Published online: 30 October 2018

\section{References}

Barley FL, Kessel D, Nicholson T, Robertson I (2006) Selective embolization of large symptomatic iatrogenic renal transplant arteriovenous fistula. Cardiovasc Intervent Radiol 29(6):1084-1087

Diaz-Buxo JA, Kopen DF, Donadio JV Jr (1974) Renal allograft arteriovenous fistula following percutaneous biopsy. J Urol 112:577-580

Eibl N, Rudolph B, Roser M, Kahl A, Mihatsch MJ, Gollasch M (2009) Arteriovenous malformation in a kidney allograft. NDT Plus 2(4):320-322

Maldonado JE, Sheps SG, Bernatz PE, Dewerd JH, Harrison EG (1964) Renal arteriovenous fistula: a reversible cause of hypertension and heart failure. Am J Med 37:499-513

Maleux G, Messiaen T, Stockx L, Vanrenterghem Y, Wilms G (2003) Transcatheter embolization of biopsy-related vascular injuries in renal allografts. Long-term technical, clinical and biochemical results. Acta Radiol 44(1):13-17

Merkus JW, Zeebregts CJ, Hoitsma AJ, Van Asten WN, Koene RA, Skotnicki SH (1993) High incidence of arteriovenous fistula after biopsy of kidney allografts. Br J Surg 80:310-312

Perini $S$ et al (1998) Transcatheter embolization of biopsy-related vascular injury in the transplant kidney: immediate and long-term outcome. J Vasc Interv Radiol 9(6):1011-1019

Rajesh GN, Sajeer K, Nair AK (2015) Device closure of pulmonary arteriovenous malformation using Amplatzer vascular plug II in hereditary hemorrhagic telangiectasia. Indian Heart J 67:455-458

Schwarz A, Hiss M, Gwinner W, Becker T, Haller H, Keberle M (2008) Course and relevance of arteriovenous fistulas after renal transplant biopsies. Am J Transplant 8:826-831

Shih CH, Liang PC, Chiang FT, Tseng CD, Tseng YZ, Hsu KL (2010) Transcatheter embolization of a huge renal arteriovenous fistula with Amplatzer vascular plug. Heart Vessel 25(4):356-358

Sundarakumar D, Kroma GM, Smith C, Lopera J, Suri R (2013) Embolization of a large high-flow renal arteriovenous fistula using 035" and 018" detachable coils. Indian J Radiol Imaging 23(2):151-154

Vij G, Hamper UM, De Jong MR, Scoutt LM (2011) A pot Pourri of abdominal Doppler cases. Sci Direct, Ultrasound Clin 6:531-550

\section{Submit your manuscript to a SpringerOpen ${ }^{\bullet}$ journal and benefit from:}

- Convenient online submission

- Rigorous peer review

- Open access: articles freely available online

High visibility within the field

- Retaining the copyright to your article

Submit your next manuscript at $>$ springeropen.com 